\title{
Interdisciplinarity: Reconciling the irreconcilable?
}

$\mathrm{T}$ he productive contributions that the biological and social sciences have made to medicine are self-evident. Research integrating these perspectives can be described as interdisciplinary (although it can also be described as multidisciplinary, transdisciplinary and cross-disciplinary, depending on the extent and nature of integration). The rise of neuroscience, epigenetics and microbiomics has created new possibilities for interdisciplinary research, just as social scientists have engaged projects that undercut the presumed divide between the social and the biological.

Interdisciplinary research is also promoted by funding bodies, including the Canadian Institutes of Health Research, and generally assumed to be valuable and beneficial. ${ }^{1}$ However, despite some very successful interdisciplinary studies, "it can hardly be said that [interdisciplinary health research] has been normalized on the campuses of Canadian universities," 2 and research combining biological and social influences remains, as a proportion, relatively rare. Why is this?

Addressing this question empirically is surprisingly difficult. Interdisciplinarity has multiple meanings; studies assess different things (journals, disciplines, authors, career trajectories) and put them into practice in different ways. Citation indices sometimes make discipline-specific assumptions that can bias against interdisciplinary work. Moreover, interdisciplinarity is judged differently between disciplines: evidence suggests its status is low in biomedical science, but average in clinical medicine and social science. These challenges are compounded by an absence of frameworks and analytic procedures to jointly evaluate different kinds of data. Moreover, they arise within academic contexts where competition is encouraged, research funding is limited and discontinuous, and coarse numerical indicators are frequently used to assess performance. Hence, in science policy, there are no general indicators of the value, extent and nature of interdisciplinarity, nor is there consensus about methods for its assessment. ${ }^{3}$

Again and again, conversation is proposed as the solution. Debate and dialogue are vital, and they can be facilitated by shared conceptions of the scale and character of the research problem and common value orientations. Small collaborative teams, good personal chemistry and physical proximity also help. Nevertheless, when it comes to collaborations between biological and social scientists, such conversations are often more troublesome than they might appear.

To illustrate, consider mental health: a field in which interdisciplinary collaboration might yield particular benefit,

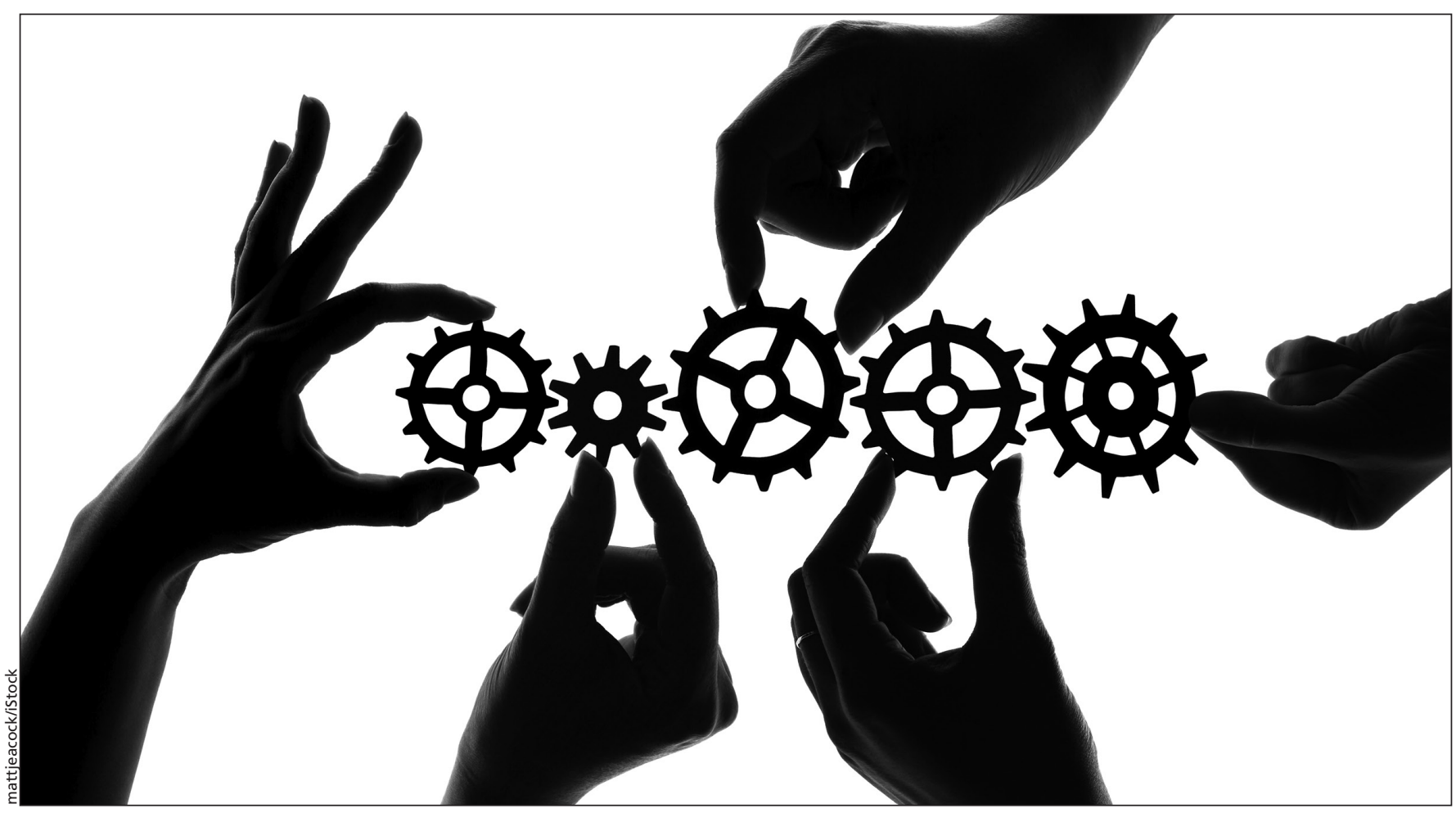


but where it is sometimes difficult to obtain even basic conceptual agreements. Biologists typically understand schizophrenia as an illness or syndrome associated with diverse neuroanatomical, physiologic and hormonal abnormalities with numerous genetic associations. However, most recognize that no consistent biomarkers for schizophrenia have been identified. On the basis of this and other evidence, many social scientists argue that schizophrenia is "real" largely to the extent that it is talked about as such. Regimes of power, knowledge and practice, associated with the hospital and the clinic, inculcated within medical training and codified in the Diagnostic and Statistical Manual of Mental Disorders and the International Classification of Diseases, have proliferated across civil society. In their wake, experiences such as voice-hearing - which might otherwise be understood as either religious, mystical phenomena or understandable responses to trauma and abuse - are instead construed as illness. Both biological and social scientists accept that these experiences occur, but the two groups conceptualize them differently. The point, here, is not that one is right and the other wrong, but that both can marshal relevant evidence.

Another issue concerns the conceptualization of social processes. Biologists mostly use what the philosopher and psychologist Rom Harre ${ }^{4}$ calls a causal metaphysic, in which outcomes are the end result of complicated, but ultimately deterministic, chains of influence. By contrast, many social scientists employ a "meaning/rule" metaphysic, whereby social processes are not so much caused as guided by shared meanings that neither impel nor determine behaviour: rather, they supply rules toward which people orient themselves.

Take for example the question of social withdrawal among people given a diagnosis of schizophrenia. Biologists might attribute social withdrawal to the cognitive and biological deficits of the syndrome, perhaps using the term "negative symptoms." Social scientists might argue that biomedical assumptions about negative symptoms are selfperpetuating, contributing to a feedback loop in which people learn to see their preferences as pathologies and interpret attempts at change as futile. How can such divergent meanings be put into practice in the same study? Doing so would presume a causal framework that many social scientists reject. Difference of this sort cannot easily be resolved.

Related to this problem is the tendency of groups to have what social scientists call emergent properties: qualities such as language that are not reducible to individuals. The delusions and auditory hallucinations often associated with schizophrenia are linked to a language that in turn reflects cultural tropes (such as religion and science fiction). Studies that simply record the presence or absence of delusions or hallucinations make social and linguistic influences largely invisible. This tends to obscure alternative explanations (e.g., childhood trauma), biasing explanations toward the biological. ${ }^{5}$

Other more specific conceptual issues may also arise. Epigenetic research into schizophrenia potentially includes both environmental and biological factors. However, in epigenetics, the environment begins at the boundary of the cell nucleus and is sometimes operationally defined only to the extent that it produces measurable biological variation at this level for example by regulating methylation. Social scientists sometimes describe this as a flattening of the environment that can conceal important nuances of social influence. Childhood trauma is associated more strongly with psychosis when there is a deliberate intent to harm, ${ }^{6}$ but this kind of relational variation cannot be meaningfully addressed within epigenetic studies that operationally define the environment in wholly biological terms.

Schizophrenia conveniently dramatizes these issues, while also suggesting what is, sometimes, at stake within them. Nevertheless, similar issues arise within any attempt to unite biological and social perspectives. Their enfolding suggests that dialogue between biological and social scientists is necessary from the very beginning of interdisciplinary research, before studies are designed. Where researchers are responding to time-bound funding calls, this is difficult; one team of bio- logical, economic and social scientists describe how it took eight years to achieve sufficient joint understanding. ${ }^{7}$

Such dialogue might be facilitated by topic-specific methodological and conceptual resources. For example, the biologist Steven Rose ${ }^{8}$ has put forward the concept of "lifelines." 8 Bayesian belief network models that incorporate conditional probabilities related to human action, and so potentially quantify something of its meaning/rule orientation, might also be valuable. More fundamentally, changes to research training and academic practice would be helpful. ${ }^{2}$

Even with such strategies, full interdisciplinarity remains a paradox. ${ }^{9}$ Both social and biological researchers are enmeshed within fields of interest where publications, grants and careers are bound up with topical, conceptual and methodological decisions. Simultaneously, the frequent recurrence of intertwined conceptual and methodological difficulties suggests that although shared work toward a common goal is possible, full epistemological unification is probably not.

\section{John Cromby BSc PhD}

School of Management, University of Leicester, Leicester UK

\section{References}

1. Whitfield K, Read C. Assumptions, ambiguities, and possibilities in interdisciplinary population health research. Can J Public Health 2004;95:434-6.

2. Hall JG, Bainbridge L, Buchan A, et al. A meeting of minds: interdisciplinary research in the health sciences in Canada. CMAJ 2006;175:763-71.

3. Huutoniemi K, Klein JT, Bruun H, et al. Analyzing interdisciplinarity: typology and indicators. Res Policy 2010;39:79-88.

4. Harre R. Cognitive science: a philosophical introduction. London (UK): Sage Publications; 2002.

5. Read J, Argyle N. Hallucinations, delusions and thought disorder among adult psychiatric inpatients with a history of child abuse. Psychiatr Serv 1999;50: 1467-72.

6. van Nierop M, Lataster T, Smeets F, et al. Psychopathological mechanisms linking childhood traumatic experiences to risk of psychotic symptoms: analysis of a large, representative populationbased sample. Schizophr Bull 2014;40(Suppl 2): S123-30.

7. Haapasaari P, Kulmala S, Kuikka S. Growing into interdisciplinarity: how to converge biology, economics, and social science in fisheries research? Ecol Soc 2012;17.

8. Rose S. Lifelines: life beyond the gene. Oxford (UK): Oxford University Press; 1997.

9. Dini P, Mehita I, Mansell R. The (im)possibility of interdisciplinarity: lessons from constructing a theoretical framework for digital ecosystems. Cult Theory Crit 2011;52:3-27.

CMAJ 2015. DOI:10.1503/cmaj.150372 